\title{
Light-quark dipole operators at the LHC
}

\author{
Eduardo da Silva Almeida, ${ }^{1, *}$ N. Rosa-Agostinho, ${ }^{2, \dagger}$ Oscar J. P. Éboli, ${ }^{1, \pitchfork}$ and M. C. Gonzalez-Garcia ${ }^{3,2,4, \S}$ \\ ${ }^{1}$ Instituto de Física, Universidade de São Paulo, 05508-090 São Paulo, Brazil \\ ${ }^{2}$ Departament de Fisica Quantica i Astrofisica and Institut de Ciencies del Cosmos, \\ Universitat de Barcelona, Diagonal 647, E-08028 Barcelona, Spain \\ ${ }^{3}$ C.N. Yang Institute for Theoretical Physics, Stony Brook University, Stony Brook, NY 11794-3849, USA \\ ${ }^{4}$ Institució Catalana de Recerca i Estudis Avancats (ICREA) Pg. Lluis Companys 23, \\ 08010 Barcelona, Spain
}

(Received 21 May 2019; published 22 July 2019)

\begin{abstract}
We study the effect of operators generating dipole couplings of the light quarks to electroweak gauge bosons on several observables at the LHC. We start by demonstrating that the determination of the gaugeboson self-couplings from electroweak diboson production at LHC is robust under the inclusion of those quark dipole operators even when let them totally unconstrained in the analysis. Conversely, we determine the bounds that the diboson data imposes on the light-quark dipole couplings and show that they represent a significant improvement over the limits arising from $Z$ and $W$ electroweak precision measurements. We also explore the sensitivity of the Drell-Yan cross section determination at LHC Run 1, and the results on resonance searches in high invariant-mass lepton pair production at Run 2 to further constrain the electroweak dipole couplings of the light quarks.
\end{abstract}

DOI: 10.1103/PhysRevD.100.013003

\section{INTRODUCTION}

The CERN LHC has provided us with invaluable information on the Standard Model (SM) such as the discovery of a Higgs boson $[1,2]$ with properties compatible with the simplest realization of electroweak symmetry breaking as well as the apparent lack of new states in the available data. In the absence of additional low-scale particles, we are compelled to describe possible deviations from the SM predictions through an effective field theory [3] containing an ordered series of higher-dimension operators built of the SM fields. In this context, and within the present experimental results, one can proceed under the assumption that the new operators are linearly invariant under the SM gauge group $S U(3)_{C} \otimes S U(2)_{L} \otimes U(1)_{Y}$, and we write

$$
\mathcal{L}_{\text {eff }}=\mathcal{L}_{\mathrm{SM}}+\sum_{n>4, j} \frac{f_{n, j}}{\Lambda^{n-4}} \mathcal{O}_{n, j}
$$

\footnotetext{
*eduardo.silva.almeida@usp.br

nuno.f.agostinho@gmail.com

*eboli@if.usp.br

${ }^{\S}$ maria.gonzalez-garcia@stonybrook.edu
}

Published by the American Physical Society under the terms of the Creative Commons Attribution 4.0 International license. Further distribution of this work must maintain attribution to the author(s) and the published article's title, journal citation, and DOI. Funded by SCOAP.
The first operators that impact the LHC physics are of $n=6$, i.e., dimension 6 . The most general dimension-6 operator basis respecting the SM gauge symmetry, as well as baryon and lepton number conservation, contains 59 independent operators, up to flavor and Hermitian conjugation $[4,5]$.

This work aims to study the operators that generate dipole couplings of the light quarks to electroweak gauge bosons that belong to the dimension- 6 operator basis, focusing on their effects on several observables at the LHC. In particular, it has been recently demonstrated that the electroweak gauge-boson couplings to fermions can impact the LHC diboson analysis [6-10] used to test the gauge-boson self-interactions. Here, we extend the analysis of these events to study their sensitivity to the inclusion of light-quark electroweak dipole couplings. The aim is twofold. First, we want to test their possible effect on the extracted information on the gauge boson selfcouplings. Second, we want to quantify the constraints that the analysis can impose on the Wilson coefficients of light-quark dipole operators. Moreover, we also explore how Drell-Yan (DY) processes can further test these operators.

The light-quark electroweak dipole operators have been previously studied using electroweak precision data (EWPD) $[11,12]$ as well as deep inelastic scattering results from HERA [12], leading to constraints on their Wilson coefficients of the order of $10 \mathrm{TeV}^{-2}$. Reference [13] includes their effect in neutral- and charge-current Drell-Yan 
production, associated production of the Higgs and a vector boson, and Higgs-boson production via vectorboson fusion at the LHC and makes an estimate of their sensitivity to be $\mathcal{O}(\mathrm{TeV})$. In the case of top quarks, the TopFitter Collaboration [14] obtained limits on the corresponding operator coefficients using mainly Run 1 data, and most recently, the SMFiT Collaboration [15] has performed a global analysis of the top-quark sector in terms of dimension- 6 operators including both Run 1 and Run 2 data and obtained bounds, which, in our notation, reach down to $\mathcal{O}\left(3 \mathrm{TeV}^{-2}\right)$ for the coefficients of the dipole operators with quarks of the third generation. Here, we show that the study of the diboson $\left(W^{+} W^{-}\right.$, $W^{ \pm} Z$ ) production at the LHC Run 1 and 2 leads to constraints on the Wilson coefficients of light-quark electroweak dipole operators that are an order of magnitude better than the ones stemming from the EWPD analysis. Finally, we compare these bounds with those originating from their contribution to the Drell-Yan process.

\section{THEORETICAL FRAMEWORK}

In this work, we extend the SM, as in Eq. (1.1), by adding dimension-6 operators that conserve $C$ and $P$ as well as lepton and baryon numbers. The basis of dimension-6 operators is not unique due to the freedom associated to the use of he equations of motion (EOM) [16-19]. Using that freedom, we choose to work in the basis of Hagiwara, Ishihara, Szalapski, and Zeppenfeld (HISZ) $[20,21]$ for the pure bosonic operators.

Our main focus is the study of operators containing electroweak dipole couplings for light quarks, which for simplicity we refer to as dipole operators in what follows. More specifically, these operators are

$$
\begin{aligned}
& \mathcal{O}_{u W, i j}=i \bar{Q}_{i} \sigma^{\mu \nu} u_{R, j} \hat{W}_{\mu \nu} \tilde{\Phi}, \quad \mathcal{O}_{u B, i j}=i \bar{Q}_{i} \sigma^{\mu \nu} u_{R, j} \hat{B}_{\mu \nu} \tilde{\Phi}, \\
& \mathcal{O}_{d W, i j}=i \bar{Q}_{i} \sigma^{\mu \nu} d_{R, j} \hat{W}_{\mu \nu} \Phi, \quad \mathcal{O}_{d B, i j}=i \bar{Q}_{i} \sigma^{\mu \nu} u_{R, j} \hat{B}_{\mu \nu} \Phi,
\end{aligned}
$$

where $\Phi$ stands for the Higgs doublet and $\tilde{\Phi}=i \sigma_{2} \Phi^{*}$. We defined $\hat{B}_{\mu \nu} \equiv i\left(g^{\prime} / 2\right) B_{\mu \nu}$ and $\hat{W}_{\mu \nu} \equiv i(g / 2) \sigma^{a} W_{\mu \nu}^{a}$, with $g$ and $g^{\prime}$ being the $S U(2)_{L}$ and $U(1)_{Y}$ gauge couplings, respectively, and $\sigma^{a}$ being the Pauli matrices. $Q$ denotes the quark doublet, $f_{R}$ are the $S U(2)_{L}$ singlet fermions, and $i, j$ are family indices.

For simplicity, we assume that the Wilson coefficient of the dipole operators are flavor diagonal and family independent; i.e., the dipole interactions are

$$
\begin{aligned}
\mathcal{L}_{\text {eff }}^{\text {DIP }}= & \frac{f_{u B}}{\Lambda^{2}} \sum_{i=1,2} \mathcal{O}_{u B, i i}+\frac{f_{u W}}{\Lambda^{2}} \sum_{i=1,2} \mathcal{O}_{u W, i i}+\frac{f_{d B}}{\Lambda^{2}} \sum_{i=1,2} \mathcal{O}_{u B, i i} \\
& +\frac{f_{d W}}{\Lambda^{2}} \sum_{i=1,2} \mathcal{O}_{u W, i i}+\text { H.c. }
\end{aligned}
$$

The effective interactions in Eq. (2.2) induce dipolelike couplings to photons, $Z$ 's and $W^{ \pm}$'s of the form

$$
\begin{aligned}
\mathcal{L}= & -\frac{e v}{\sqrt{2}}\left[\frac{F_{f \gamma}}{\Lambda^{2}} \bar{f} \sigma^{\mu \nu} f \partial_{\mu} A_{\nu}+\frac{F_{f Z}}{\Lambda^{2}} \bar{f} \sigma^{\mu \nu} f \partial_{\mu} Z_{\nu}\right] \\
& -e v\left[\bar{f} \sigma^{\mu \nu}\left(\frac{F_{f f^{\prime} W}^{L}}{\Lambda^{2}} P_{L}+\frac{F_{f f^{\prime} W}^{R}}{\Lambda^{2}} P_{R}\right) f^{\prime} \partial_{\mu} W_{\nu}^{+}+\text {H.c. }\right],
\end{aligned}
$$

where $P_{L(R)}$ is the left- (right-)handed chiral projector and

$$
\begin{aligned}
\frac{F_{u \gamma}}{\Lambda^{2}} & =\frac{f_{u W}}{\Lambda^{2}}+\frac{f_{u B}}{\Lambda^{2}}, \quad \frac{F_{u Z}}{\Lambda^{2}}=\frac{c_{W}}{s_{W}} \frac{f_{u W}}{\Lambda^{2}}-\frac{s_{W}}{c_{W}} \frac{f_{u B}}{\Lambda^{2}}, \\
\frac{F_{d \gamma}}{\Lambda^{2}} & =\frac{f_{d W}}{\Lambda^{2}}-\frac{f_{d B}}{\Lambda^{2}}, \quad \frac{F_{d Z}}{\Lambda^{2}}=\frac{c_{W}}{s_{W}} \frac{f_{d W}}{\Lambda^{2}}+\frac{s_{W}}{c_{W}} \frac{f_{d B}}{\Lambda^{2}}, \\
\frac{F_{u d W}^{R}}{\Lambda^{2}} & =\frac{1}{s_{W}} \frac{f_{u W}}{\Lambda^{2}}, \quad \frac{F_{u d W}^{L}}{\Lambda^{2}}=\frac{1}{s_{W}} \frac{f_{d W}}{\Lambda^{2}} .
\end{aligned}
$$

Here, $s_{W}\left(c_{W}\right)$ is the sine (cosine) of the weak mixing angle.

Consequently, the electroweak dipole operators contribute to any process at the LHC initiated by the quark and antiquark components of the colliding protons. In particular, they take part in electroweak diboson production $p p \rightarrow W^{+} W^{-}$and $p p \rightarrow Z W^{ \pm}$. Notwithstanding, there are further dimension- 6 operators that contribute to these processes like the following additional operators that change the couplings between gauge bosons and fermions,

$$
\begin{aligned}
\mathcal{O}_{\Phi L, i j}^{(1)} & =\Phi^{\dagger}\left(i \stackrel{\leftrightarrow}{D_{\mu}} \Phi\right)\left(\bar{L}_{i} \gamma^{\mu} L_{j}\right), \\
\mathcal{O}_{\Phi L, i j}^{(3)} & =\Phi^{\dagger}\left(i{\stackrel{\leftrightarrow}{D^{a}}}_{\mu} \Phi\right)\left(\bar{L}_{i} \gamma^{\mu} T_{a} L_{j}\right), \\
\mathcal{O}_{\Phi Q, i j}^{(1)} & =\Phi^{\dagger}\left(i \stackrel{\leftrightarrow}{D}_{\mu} \Phi\right)\left(\bar{Q}_{i} \gamma^{\mu} Q_{j}\right), \\
\mathcal{O}_{\Phi Q, i j}^{(3)} & =\Phi^{\dagger}\left(i \stackrel{\leftrightarrow}{D^{a}}{ }_{\mu} \Phi\right)\left(\bar{Q}_{i} \gamma^{\mu} T_{a} Q_{j}\right), \\
\mathcal{O}_{\Phi u, i j}^{(1)} & =\Phi^{\dagger}\left(i \stackrel{\leftrightarrow}{D}_{\mu} \Phi\right)\left(\bar{u}_{R_{i}} \gamma^{\mu} u_{R_{j}}\right), \\
\mathcal{O}_{\Phi d, i j}^{(1)} & =\Phi^{\dagger}\left(i \stackrel{\leftrightarrow}{D}_{\mu} \Phi\right)\left(\bar{d}_{R_{i}} \gamma^{\mu} d_{R_{j}}\right), \\
\mathcal{O}_{\Phi e, i j}^{(1)} & =\Phi^{\dagger}\left(i \stackrel{\leftrightarrow}{D}_{\mu} \Phi\right)\left(\bar{e}_{R_{i}} \gamma^{\mu} e_{R_{j}}\right), \\
\mathcal{O}_{\Phi u d}^{(1)} & =\tilde{\Phi}^{\dagger}\left(i \stackrel{\leftrightarrow}{D_{\mu}} \Phi\right)\left(\bar{u}_{R} \gamma^{\mu} d_{R}+\text { H.c. }\right),
\end{aligned}
$$

where the lepton doublet (singlet) is denoted by $L(e)$ and we defined $\Phi^{\dagger} \stackrel{\leftrightarrow}{D}_{\mu} \Phi=\Phi^{\dagger} D_{\mu} \Phi-\left(D_{\mu} \Phi\right)^{\dagger} \Phi$ and $\Phi^{\dagger}{\stackrel{\leftrightarrow}{D^{a}}}_{\mu} \Phi=$ $\Phi^{\dagger} T^{a} D_{\mu} \Phi-\left(D_{\mu} \Phi\right)^{\dagger} T^{a} \Phi$ with $T^{a}=\sigma^{a} / 2$.

In addition to the above fermionic operators, diboson production also involves triple gauge couplings (TGCs), which are directly modified by one operator that contains exclusively gauge bosons, 


$$
\mathcal{O}_{W W W}=\operatorname{Tr}\left[\hat{W}_{\mu}^{\nu} \hat{W}_{\nu}^{\rho} \hat{W}_{\rho}^{\mu}\right],
$$

as well as three additional dimension-6 operators that include Higgs and electroweak gauge fields in the HISZ basis

$$
\begin{aligned}
\mathcal{O}_{W} & =\left(D_{\mu} \Phi\right)^{\dagger} \hat{W}^{\mu \nu}\left(D_{\nu} \Phi\right), \\
\mathcal{O}_{B} & =\left(D_{\mu} \Phi\right)^{\dagger} \hat{B}^{\mu \nu}\left(D_{\nu} \Phi\right) \quad \text { and } \\
\mathcal{O}_{B W} & =\Phi^{\dagger} \hat{B}_{\mu \nu} \hat{W}^{\mu \nu} \Phi .
\end{aligned}
$$

It is interesting to notice that, besides giving a direct contribution to TGCs, $\mathcal{O}_{B W}$ also leads to a finite renormalization of the SM gauge fields, therefore modifying the electroweak gauge-boson couplings. Furthermore, two other dimension- 6 operators also enter in our studies via finite renormalization effects, namely,

$$
\begin{aligned}
\mathcal{O}_{L L L L} & =\left(\bar{L} \gamma^{\mu} L\right)\left(\bar{L} \gamma^{\mu} L\right) \quad \text { and } \\
\mathcal{O}_{\Phi, 1} & =\left(D_{\mu} \Phi\right)^{\dagger} \Phi \Phi^{\dagger}\left(D^{\mu} \Phi\right) .
\end{aligned}
$$

In brief, $\mathcal{O}_{L L L L}$ gives a finite correction to the Fermi constant, while $\mathcal{O}_{B W}$ and $\mathcal{O}_{\Phi, 1}$ lead to a finite correction of the $S$ and $T$ oblique parameters, respectively.

At this point, we still have redundant operators in our basis. To eliminate two blind directions $[22,23]$ that appear in the EWPD analysis, we use the freedom associated to the use of EOM to remove the operator combinations [24]

$$
\sum_{i} \mathcal{O}_{\Phi L, i i}^{(1)} \text { and } \sum_{i} \mathcal{O}_{\Phi L, i i}^{(3)}
$$

from our operator basis. As we assume no family mixing and the Wilson coefficients to be generation independent, the dimension- 6 contributions to the electroweak diboson data (EWDBD) depend upon 16 Wilson coefficients, namely,

$$
\begin{aligned}
\mathcal{L}_{\text {eff }}^{\text {EWDBD }}= & \frac{f_{\Phi Q}^{(1)}}{\Lambda^{2}} \sum_{i=1,2,3} \mathcal{O}_{\Phi Q, i i}^{(1)}+\frac{f_{\Phi Q}^{(3)}}{\Lambda^{2}} \sum_{i=1,2,3} \mathcal{O}_{\Phi Q, i i}^{(3)} \\
& +\frac{f_{\Phi u}^{(1)}}{\Lambda^{2}} \sum_{i=1,2} \mathcal{O}_{\Phi u, i i}^{(1)}+\frac{f_{\Phi d}^{(1)}}{\Lambda^{2}} \sum_{i=1,2,3} \mathcal{O}_{\Phi d, i i}^{(1)} \\
& +\frac{f_{\Phi e}^{(1)}}{\Lambda^{2}} \sum_{i=1,2,3} \mathcal{O}_{\Phi e, i i}^{(1)}+\frac{f_{W}}{\Lambda^{2}} \mathcal{O}_{W}+\frac{f_{B}}{\Lambda^{2}} \mathcal{O}_{B} \\
& +\frac{f_{W W W}}{\Lambda^{2}} \mathcal{O}_{W W W}+\frac{f_{B W}}{\Lambda^{2}} \mathcal{O}_{B W}+\frac{f_{\Phi, 1}}{\Lambda^{2}} \mathcal{O}_{\Phi, 1} \\
& +\frac{f_{L L L L}}{\Lambda^{2}} \mathcal{O}_{L L L L}+\frac{f_{\Phi u d}^{(1)}}{\Lambda^{2}} \sum_{i=1,2} \mathcal{O}_{\Phi u d, i i}^{(1)}+\mathcal{L}_{\mathrm{eff}}^{\mathrm{DIP}} .
\end{aligned}
$$

In the limit of vanishing light-quark masses, the dipole operators contribute to different diboson helicity amplitudes than the SM or any of the other operators in Eq. (2.10) (more below) due to their tensor structure. Therefore, there is no interference between the contributions coming from the dipole operators and the SM and other dimension- 6 operators in Eq. (2.10). Consequently, the dipole operators only contribute to these observables at the quadratic level. For the same reason, $\mathcal{O}_{\Phi u d}^{(1)}$, which modifies the couplings of $W$ 's to right-handed quark pairs, does not interfere with the SM contributions nor with the other fermionic operators.

Since the light-quark dipole operators modify the $Z$ and $W^{ \pm}$couplings, they can be constrained by the EWPD observables [11], which altogether receive corrections from a subset of 13 operators,

$$
\begin{aligned}
\mathcal{L}_{\text {eff }}^{\text {EWPD }}= & \frac{f_{\Phi Q}^{(1)}}{\Lambda^{2}} \sum_{i=1,2,3} \mathcal{O}_{\Phi Q, i i}^{(1)}+\frac{f_{\Phi Q}^{(3)}}{\Lambda^{2}} \sum_{i=1,2,3} \mathcal{O}_{\Phi Q, i i}^{(3)} \\
& +\frac{f_{\Phi u}^{(1)}}{\Lambda^{2}} \sum_{i=1,2} \mathcal{O}_{\Phi u, i i}^{(1)}+\frac{f_{\Phi d}^{(1)}}{\Lambda^{2}} \sum_{i=1,2,3} \mathcal{O}_{\Phi d, i i}^{(1)} \\
& +\frac{f_{\Phi e}^{(1)}}{\Lambda^{2}} \sum_{i=1,2,3} \mathcal{O}_{\Phi e, i i}^{(1)}+\frac{f_{B W}}{\Lambda^{2}} \mathcal{O}_{B W}+\frac{f_{\Phi, 1}}{\Lambda^{2}} \mathcal{O}_{\Phi, 1} \\
& +\frac{f_{L L L L}}{\Lambda^{2}} \mathcal{O}_{L L L L}+\frac{f_{\Phi u d}^{(1)}}{\Lambda^{2}} \sum_{i=1,2} \mathcal{O}_{\Phi u d, i i}^{(1)}+\mathcal{L}_{\mathrm{eff}}^{\mathrm{DIP}},
\end{aligned}
$$

where, due to the arguments above, the eight operators in the first two lines contribute linearly to the EWPD observables, see Ref. [25], while the five in the last line enter only at quadratic order. Indeed, the contributions of the dipole operators to the decay widths of the weak gauge bosons are

$$
\begin{aligned}
& \frac{\Delta \Gamma_{f f}^{Z}}{\Gamma_{f f}^{Z}}=\frac{1}{g_{L}^{f 2}+g_{R}^{f 2}} \frac{e^{2} v^{4}}{8 \Lambda^{4}}\left|F_{f Z}\right|^{2}, \\
& \frac{\Delta \Gamma_{u d}^{W}}{\Gamma_{u d}^{W}}=\frac{e^{2} v^{4}}{4 \Lambda^{4}}\left(\left|F_{u d W}^{L}\right|^{2}+\left|F_{u d W}^{R}\right|^{2}\right),
\end{aligned}
$$

where $g_{L}^{f}=T_{3}^{f}-Q_{f} s_{W}^{2}$ and $g_{L}^{f}=-Q_{f} s_{W}^{2}$ are the usual SM couplings.

\section{EFFECTS IN ELECTROWEAK DIBOSON PRODUCTION}

Deviations of TGC and gauge-boson interactions with quarks from the SM ones modify the high-energy behavior of the scattering of quark pairs into two electroweak gauge bosons since such anomalous interactions can spoil the cancellations built in the SM. For the $W^{+} W^{-}$and $W^{ \pm} Z$ 
channels, the leading scattering amplitudes in the helicity basis receive contributions from 12 of the 16 operators in Eq. (2.10) $[25,26]$,

$$
\begin{aligned}
& A\left(d_{-} \bar{d}_{+} \rightarrow W_{0}^{+} W_{0}^{-}\right) \\
& =i \frac{s}{\Lambda^{2}} \sin \theta\left\{-\frac{g^{2}}{24 c_{W}^{2}}\left(3 c_{W}^{2} f_{W}-s_{W}^{2} f_{B}\right)\right. \\
& \left.+\frac{1}{4}\left(f_{\Phi Q}^{(3)}-4 f_{\Phi Q}^{(1)}\right)\right\} \text {, } \\
& A\left(d_{-} \bar{d}_{+} \rightarrow W_{ \pm}^{+} W_{ \pm}^{-}\right)=-i \frac{s}{\Lambda^{2}} \sin \theta \frac{3 g^{4}}{8} f_{W W W}, \\
& A\left(d_{+} \bar{d}_{-} \rightarrow W_{0}^{+} W_{0}^{-}\right)=-i \frac{s}{\Lambda^{2}} \sin \theta\left\{\frac{g^{2} s_{W}^{2}}{12 c_{W}^{2}} f_{B}+f_{\Phi d}^{(1)}\right\}, \\
& A\left(u_{-} \bar{u}_{+} \rightarrow W_{0}^{+} W_{0}^{-}\right) \\
& =i \frac{s}{\Lambda^{2}} \sin \theta\left\{\frac{g^{2}}{24 c_{W}^{2}}\left(3 c_{W}^{2} f_{W}+s_{W}^{2} f_{B}\right)\right. \\
& \left.-\frac{1}{4}\left(f_{\Phi Q}^{(3)}+4 f_{\Phi Q}^{(1)}\right)\right\} \\
& A\left(u_{+} \bar{u}_{-} \rightarrow W_{0}^{+} W_{0}^{-}\right)=i \frac{s}{\Lambda^{2}} \sin \theta\left\{\frac{g^{2} s_{W}^{2}}{6 c_{W}^{2}} f_{B}-f_{\Phi u}^{(1)}\right\}, \\
& A\left(d_{-} \bar{u}_{+} \rightarrow Z_{ \pm} W_{ \pm}^{-}\right)=i \frac{s}{\Lambda^{2}} \sin \theta \frac{3 c_{W} g^{4}}{4 \sqrt{2}} f_{W W W}, \\
& A\left(d_{-} \bar{u}_{+} \rightarrow W_{0}^{-} Z_{0}\right)=i \frac{s}{\Lambda^{2}} \sin \theta\left\{\frac{g^{2}}{4 \sqrt{2}} f_{W}-\frac{1}{2 \sqrt{2}} f_{\Phi Q}^{(3)}\right\}, \\
& A\left(d_{+} \bar{u}_{-} \rightarrow W_{0}^{-} Z_{0}\right)=-i \frac{s}{\Lambda^{2}} \sin \theta \sqrt{2} f_{\text {Фud }}^{(1)}, \\
& A\left(d_{-} \bar{d}_{-} \rightarrow W_{0}^{+} W_{+}^{-}\right)=-A\left(d_{+} \bar{d}_{+} \rightarrow W_{-}^{+} W_{0}^{-}\right) \\
& =-\frac{s}{\Lambda^{2}} \sin \theta g f_{d W} \\
& \left.=-\frac{s}{\Lambda^{2}} \sin \theta e\left(s_{W} F_{d \gamma}+c_{W} F_{d Z}\right)\right), \\
& A\left(u_{-} \bar{u}_{-} \rightarrow W_{0}^{+} W_{+}^{-}\right)=-A\left(u_{+} \bar{u}_{+} \rightarrow W_{-}^{+} W_{0}^{-}\right) \\
& =-\frac{s}{\Lambda^{2}} \sin \theta g f_{u W} \\
& \left.=-\frac{s}{\Lambda^{2}} \sin \theta e\left(s_{W} F_{u \gamma}+c_{W} F_{u Z}\right)\right),
\end{aligned}
$$

$$
\begin{gathered}
\begin{aligned}
A\left(d_{-} \bar{u}_{-} \rightarrow W_{0}^{-} Z_{+}\right) & =\frac{s}{\Lambda^{2}} \sin \theta \frac{g}{\sqrt{2} c_{W}}\left\{s_{W}^{2} f_{u B}+c_{W}^{2} f_{u W}\right\} \\
= & \frac{s}{\Lambda^{2}} \sin \theta \frac{e}{\sqrt{2}}\left\{2 c_{W} F_{u d W}^{R}-F_{u Z}\right\} \\
A\left(d_{-} \bar{u}_{-} \rightarrow W_{+}^{-} Z_{0}\right) & =-\frac{s}{\Lambda^{2}} \sin \theta \frac{g}{\sqrt{2}} f_{u W} \\
& =-\frac{s}{\Lambda^{2}} \sin \theta \frac{e}{\sqrt{2}} F_{u d W}^{R}, \\
A\left(d_{+} \bar{u}_{+} \rightarrow W_{0}^{-} Z_{-}\right)= & -\frac{s}{\Lambda^{2}} \sin \theta \frac{g}{\sqrt{2} c_{W}}\left\{s_{W}^{2} f_{d B}-c_{W}^{2} f_{d W}\right\} \\
= & \frac{s}{\Lambda^{2}} \sin \theta \frac{e}{\sqrt{2}}\left\{2 c_{W} F_{u d W}^{L}-F_{d Z}\right\}
\end{aligned} \\
A\left(d_{+} \bar{u}_{+} \rightarrow W_{+}^{-} Z_{0}\right)=-\frac{s .11)}{\Lambda^{2}} \sin \theta \frac{g}{\sqrt{2}} f_{d W} \\
=-\frac{s}{\Lambda^{2}} \sin \theta \frac{e}{\sqrt{2}} F_{u d W}^{L},
\end{gathered}
$$

where $s$ stands for the center-of-mass energy and $\theta$ is the polar angle in the center-of-mass frame. In Eqs. (3.9)(3.14), we give the contribution from the dipole operators to the helicity amplitudes at high energy and explicitly show that they contribute to helicity configurations different from that of any other operator as mentioned in the previous section. Also, for convenience, in the last equality of those equations, we give the expression in terms of the effective couplings in Eq. (2.3).

Diboson production has been used by the LHC collaborations to directly scrutinize the structure of the electroweak triple gauge-boson coupling well beyond the sensitivity reached at LEP2 [27]. In particular, both ATLAS and CMS collaborations have used their full data samples from Run 1 of LHC on $W^{+} W^{-}[28,29]$ and $W^{ \pm} Z$ $[30,31]$ productions to constrain the possible deviations of TGCs from the SM structure. For Run 2, the number of experimental studies aiming at deriving the corresponding limits is still rather sparse [32]. However, as described in Ref. [9], one can use the published ATLAS results on $W^{ \pm} Z$ [33] and $W^{+} W^{-}$[34] productions with $36.1 \mathrm{fb}^{-1}$ to study TGCs.

Altogether, we perform an analysis aimed at constraining the Wilson coefficients in the effective Lagrangian in Eq. (2.10) using the data on $W^{+} W^{-}$and $W^{ \pm} Z$ productions in the leptonic channel. In particular, we include the available kinematic distributions most sensitive for TGC analysis, which are as follows: 


\begin{tabular}{lcccc}
\hline Channel $(a)$ & Distribution & No. of bins & Dataset & Int Lum \\
\hline$W W \rightarrow \ell^{+} \ell^{\prime-}+/ E_{T}(0 j)$ & $p_{T}^{\text {leading,lepton }}$ & 3 & ATLAS 8 TeV, & $20.3 \mathrm{fb}^{-1}[28]$ \\
$W W \rightarrow \ell^{+} \ell^{(\prime)}+/ E_{T}(0 j)$ & $m_{\ell \ell^{(\prime)}}$ & 8 & CMS 8 TeV, & $19.4 \mathrm{fb}^{-1}[29]$ \\
$W Z \rightarrow \ell^{+} \ell^{-} \ell^{(\prime) \pm}$ & $m_{T}^{W Z}$ & 6 & ATLAS 8 TeV, & $20.3 \mathrm{fb}^{-1}[30]$ \\
$W Z \rightarrow \ell^{+} \ell^{-} \ell^{(\prime) \pm}+/ E_{T}$ & $Z$ candidate $p_{T}^{\ell \ell}$ & 10 & CMS 8 TeV, & $19.6 \mathrm{fb}^{-1}[31]$ \\
$W W \rightarrow e^{ \pm} \mu^{\mp}+/ E_{T}(0 j)$ & $m_{T}$ & 17 & ATLAS 13 TeV, & $36.1 \mathrm{fb}^{-1}[34]$ \\
$W Z \rightarrow \ell^{+} \ell^{-} \ell^{(\prime) \pm}$ & $m_{T}^{W Z}$ & 6 & ATLAS 13 TeV, & $36.1 \mathrm{fb}^{-1}[33]$ \\
\hline
\end{tabular}

For details of the analysis of the EWDBD from Run 1 and Run 2, we refer the reader to Refs. [8,9], which contain our procedure, as well as its validation against the TGC results from the experimental collaborations. In brief, the procedure to obtain the prediction of the relevant kinematical distributions in the presence of the dimension- 6 operators is as follows. We simulate $W^{+} W^{-}$and $W^{ \pm} Z$ events within the experimental fiducial regions by applying the same cuts and isolation criteria adopted by the corresponding experimental analysis. This is carried out by using MADGRAPH5 [35] with the UFO files for our effective Lagrangian generated with FEYNRULES [36,37]. We employ PyTHIA6.4 [38] to perform the parton shower, while the fast detector simulation is done with DELPHES
[39]. To account for higher-order corrections and additional detector effects, we simulate the corresponding SM $W^{+} W^{-}$ and $W^{ \pm} Z$ events and normalize our results bin by bin to the SM predictions provided by the experimental collaborations. Then, we apply these correction factors to our simulated $W V$ distributions in the presence of the anomalous couplings.

The statistical confrontation of these predictions with the LHC data is made by means of a binned log-likelihood function based on the contents of the different bins in the kinematical distribution of each channel. Besides the statistical errors, we incorporate the systematic and theoretical uncertainties, including also their corresponding correlations. With this, we build $\chi_{\text {EWDBD }}^{2}$,

$$
\chi_{\mathrm{EWDBD}}^{2}\left(f_{B}, f_{W}, f_{W W W}, f_{B W}, f_{\Phi, 1}, f_{\Phi, Q}^{(3)}, f_{\Phi, Q}^{(1)}, f_{\Phi, u}^{(1)}, f_{\Phi, d}^{(1)}, f_{\Phi, u d}^{(1)}, f_{\Phi, e}^{(1)}, f_{L L L L}, f_{u B}, f_{u W}, f_{d B}, f_{d W}\right)
$$

To this, we want to add the information from EWPD, in particular from $Z$ and $W$ pole measurements. We do so by constructing a $\chi^{2}$ function,

$$
\chi_{\mathrm{EWPD}}^{2}\left(f_{B W}, f_{\Phi, 1}, f_{\Phi, Q}^{(3)}, f_{\Phi, Q}^{(1)}, f_{\Phi, u}^{(1)}, f_{\Phi, d}^{(1)}, f_{\Phi, u d}^{(1)}, f_{\Phi, e}^{(1)}, f_{L L L L}, f_{u B}, f_{u W}, f_{d B}, f_{d W}\right),
$$

including 15 observables, 12 of which are $Z$ observables [40],

$$
\Gamma_{Z}, \quad \sigma_{h}^{0}, \quad \mathcal{A}_{\ell}\left(\tau^{\mathrm{pol}}\right), \quad R_{\ell}^{0}, \quad \mathcal{A}_{\ell}(\mathrm{SLD}), \quad A_{\mathrm{FB}}^{0, l}, \quad R_{c}^{0}, \quad R_{b}^{0}, \quad \mathcal{A}_{c}, \quad \mathcal{A}_{b}, \quad A_{\mathrm{FB}}^{0, c}, \quad \text { and } \quad A_{\mathrm{FB}}^{0, b},
$$

and three are $W$ observables: $M_{W}$ [41], $\Gamma_{W}$ [42], and $\operatorname{Br}(W \rightarrow \ell \nu)$ [41]. We compare those with the corresponding predictions including the effect of all operators in Eq. (2.11). In building $\chi_{\text {EWPD }}^{2}$, we incorporate the correlations among the above observables from Ref. [40] and the SM predictions and their uncertainties from Ref. [43].

To single out the possible effect of the dipole operators in the EWDBD analysis, we make a combined analysis in which we include their effect in EWPBD but not in the EWPD observables. This is, we make a fit to EWDBD + EWPD in terms of 16 operator coefficients using

$$
\begin{aligned}
\chi_{\mathrm{EWDBD}+\mathrm{EWPD}}^{2}\left(f_{B}, f_{W}, f_{W W W}, f_{B W}, f_{\Phi, 1}, f_{\Phi, Q}^{(3)}, f_{\Phi, Q}^{(1)}, f_{\Phi, u}^{(1)}, f_{\Phi, d}^{(1)}, f_{\Phi, u d}^{(1)}, f_{\Phi, e}^{(1)}, f_{L L L L}, f_{u B}, f_{u W}, f_{d B}, f_{d W}\right) \\
=\chi_{\mathrm{EWDBD}}^{2}\left(f_{B}, f_{W}, f_{W W W}, f_{B W}, f_{\Phi, 1}, f_{\Phi, Q}^{(3)}, f_{\Phi, Q}^{(1)}, f_{\Phi, u}^{(1)}, f_{\Phi, d}^{(1)}, f_{\Phi, u d}^{(1)}, f_{\Phi, e}^{(1)}, f_{L L L L}, f_{u B}, f_{u W}, f_{d B}, f_{d W}\right) \\
\quad+\chi_{\mathrm{EWPD}}^{2}\left(f_{B W}, f_{\Phi, 1}, f_{\Phi, Q}^{(3)}, f_{\Phi, Q}^{(1)}, f_{\Phi, u}^{(1)}, f_{\Phi, d}^{(1)}, f_{\Phi, u d}^{(1)}, f_{\Phi, e}^{(1)}, f_{L L L L}, f_{u B}=0, f_{u W}=0, f_{d B}=0, f_{d W}=0\right) .
\end{aligned}
$$

We then compare the allowed parameter ranges for the coefficients with those obtained from the a combined analysis in which the dipole operators are set to zero in the analysis of EWDBD as well. 


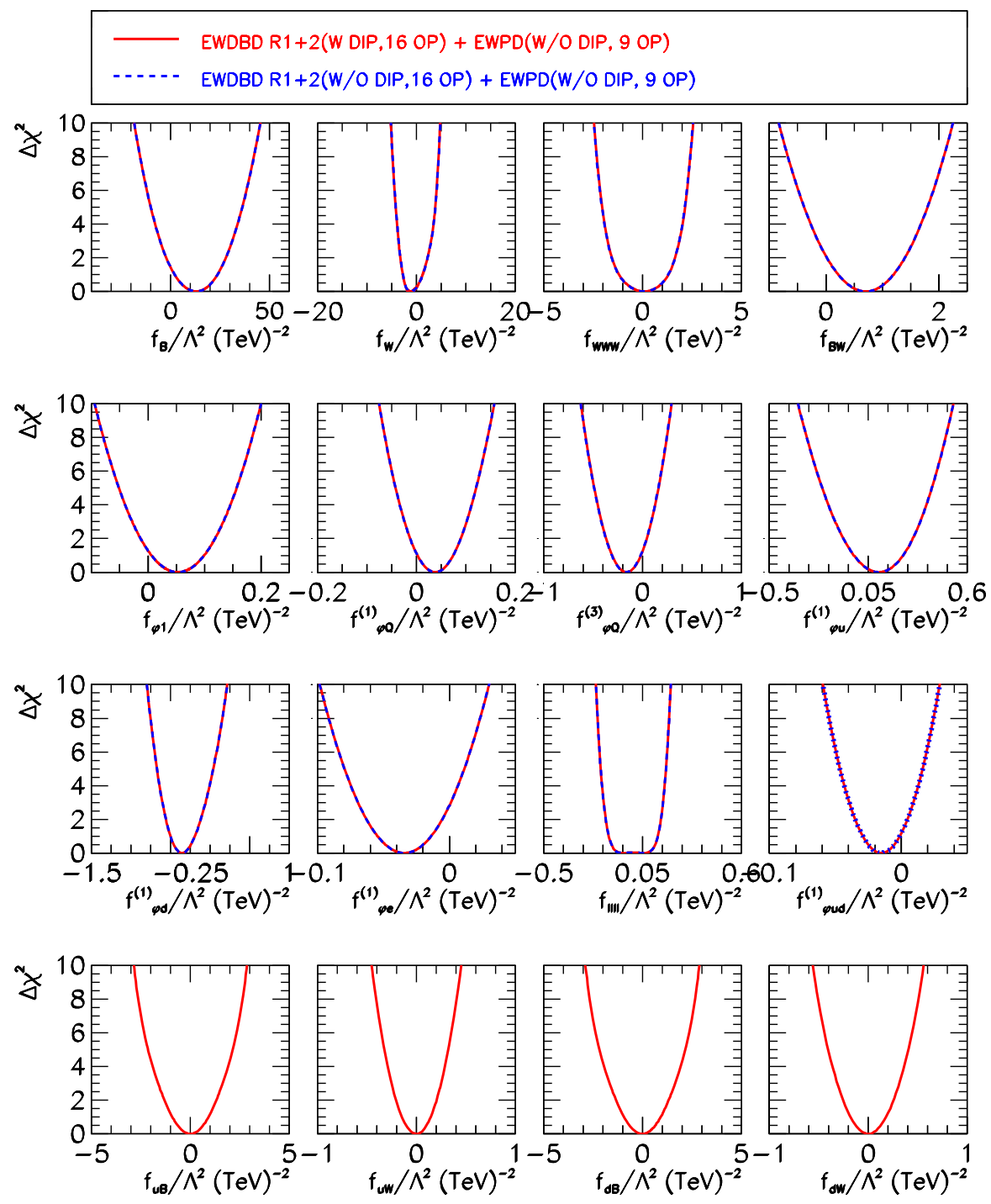

FIG. 1. $\Delta \chi^{2}$ dependence on the Wilson coefficients of 16 operators (after marginalization in each panel over the undisplayed ones) entering the analysis of EWDBD from LHC Run 1 and 2 in combination with the EWPD. The red line includes the effect of light-quark dipole operators in diboson production but does not include them in the EWPD $\chi^{2}$ [see Eq. (3.17)]. The blue dashed lines are the results of the corresponding analysis without including the light-quark dipole operators (see the text for details).

The results of these analyses are shown in Fig. 1, in which we display one-dimensional projections of the $\Delta \chi^{2}$ for both analyses. In each panel, $\chi^{2}$ has been marginalized over all 15 (or 11) other coefficients. This figure clearly illustrates that the constraints on the 12 coefficients $f_{B}, f_{W}$, $f_{W W W}, f_{B W}, f_{\Phi, 1}, f_{\Phi, Q}^{(3)}, f_{\Phi, Q}^{(1)}, f_{\Phi, u}^{(1)}, f_{\Phi, d}^{(1)}, f_{\Phi, u d}^{(1)}, f_{\Phi, e}^{(1)}$, and $f_{L L L L}$ from the combined analysis of EWDBD at LHC and EWPD are robust independently of the inclusion of the dipole operators in the analysis.

\section{A. Comparison with EWPD bounds}

Figure 1 also illustrates the power of the high-energy LHC data in diboson gauge production to impose severe constraints on the electroweak dipole couplings of the light quarks. We quantify how much these bounds improve over the ones from EWPD in Fig. 2, in which where we show in the red line the constraints from the above analysis together with those obtained from $Z$ - and $W$-pole EWPD exclusively (black lines). To estimate the dependence of the EWPD bounds on the presence of other operators contributing to those observables, we show also the results when the coefficients of all nondipole operators are set to zero (the black dashed line in Fig. 2). As expected, the bounds are only a bit stronger [about a factor $\mathcal{O}(30 \%)$ ] when no other contribution is included. As mentioned above, dipole operators enter quadratically in EWDP observables, so their contribution cannot cancel against that of any other dimension- 6 operator. The bounds derived, however, assume that there will be no cancellation against possible effects of dimension-8 operators. 


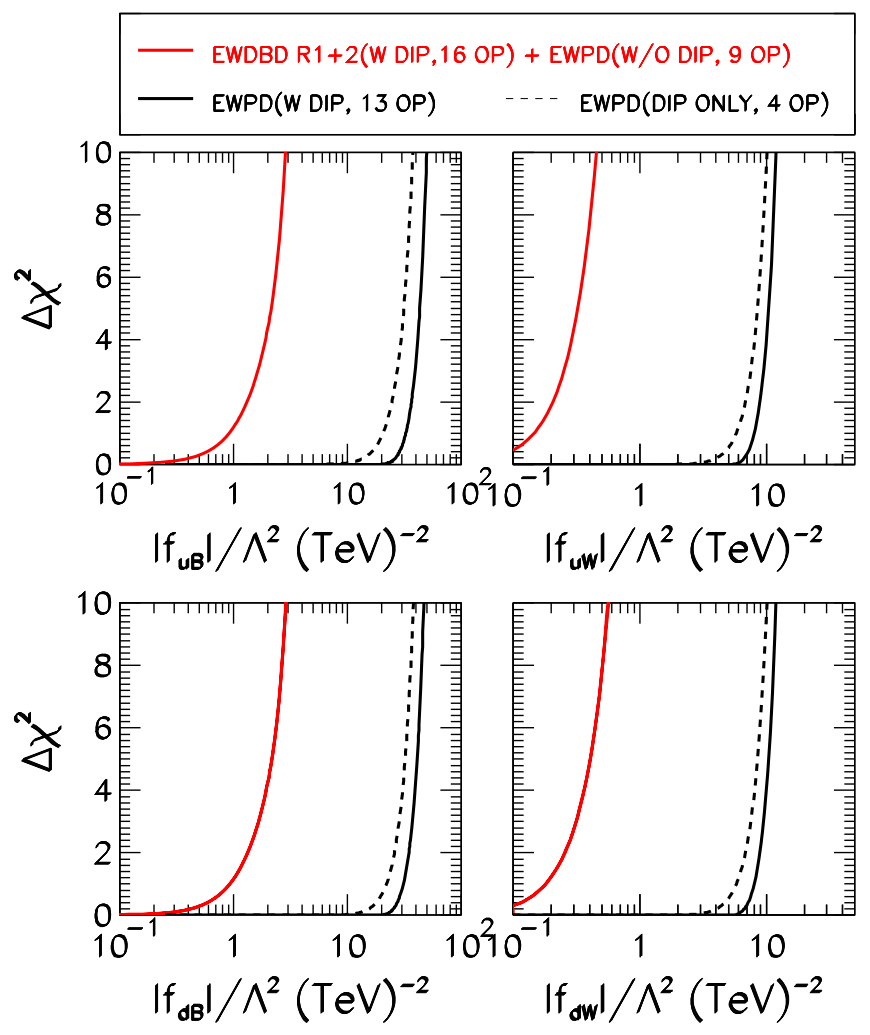

FIG. 2. $\Delta \chi^{2}$ dependence on the four fermionic Wilson coefficients of the dipole operators from the combined analysis of diboson data and EWPD after marginalizing over the 15 undisplayed coefficients (full red lines), EWPD after marginalizing over the 12 undisplayed parameters (full black lines) and EWPD with only the four-quark dipole operator after marginalizing over the three undisplayed coefficients (black dashed line).

As seen in Fig. 2, the bounds from EWPD are weaker than those from LHC EWDBD by more than an order of magnitude. The reason for this is twofold. First, the EWPD constraints are mainly driven by the $Z$ hadronic observables, which bound the combinations $F_{q Z} / \Lambda^{2}$ in Eq. (2.3), which means that there is a large degeneracy between the constraints on $f_{q W} / \Lambda^{2}$ and $\tan ^{2} \theta_{W} f_{q B} / \Lambda^{2}$. The degeneracy is broken only by the data on the $W$ width, which is much less precisely known. Second, the contributions from dipole operators to EWDBD grow as $s$, as seen in Eqs. (3.9)-(3.14) and hence the power of the high-energy LHC data to constrain them.

These behaviors are explicitly displayed in Fig. 3, in which we show the strong correlations in the $95 \%$ allowed region from the EWPD analysis in the plane $f_{q W} / \Lambda^{2}$ vs $f_{q B} / \Lambda^{2}$. We also present in this figure the corresponding allowed region on the plane $F_{q Z} / \Lambda^{2} v s F_{q \gamma} / \Lambda^{2}$ from which we can see that the EWPD bounds on the electric dipole coupling $F_{q \gamma} / \Lambda^{2}$ are visibly weaker. On the other hand, as seen in Eqs. (3.9)-(3.14), the production of pairs of electroweak gauge bosons receives independent contributions from different combinations of the effective light-quark dipole couplings to $Z, W$, and $\gamma$. Therefore, the inclusion of the EWDBD in the analysis helps constraining both the $\mathrm{Z}$ and $\gamma$ dipole couplings with similar precision. This is explicitly shown in Fig. 4, in which we plot the $95 \%$ allowed region from the EWDBD analysis in both planes $f_{q W} / \Lambda^{2}$ vs $f_{q B} / \Lambda^{2}$ and $F_{q Z} / \Lambda^{2}$ vs $F_{q} \gamma / \Lambda^{2}$.

\section{EFFECTS IN DRELL-YAN PRODUCTION}

One of the cleanest processes at LHC is the Drell-Yan production of pairs of high invariant-mass electrons and muons. Light-quark dipole operators contribute to this process in the production vertex of the intermediate $Z$ and $\gamma$ and hence can be constrained by these precise LHC results. Furthermore, the dipole operators also lead
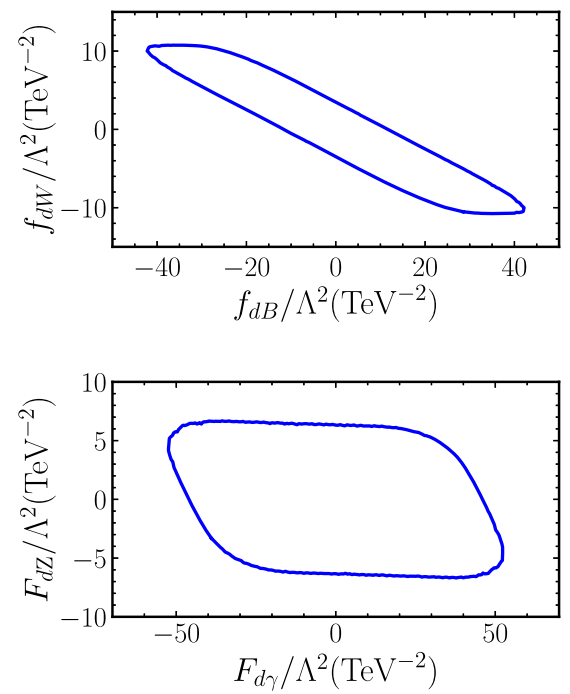

FIG. 3. $95 \%$ allowed region from the EWPD analysis in planes $f_{q W} / \Lambda^{2}$ vs $f_{q B} / \Lambda^{2}$ and $F_{q Z} / \Lambda^{2}$ vs $F_{q} \gamma / \Lambda^{2}$. 

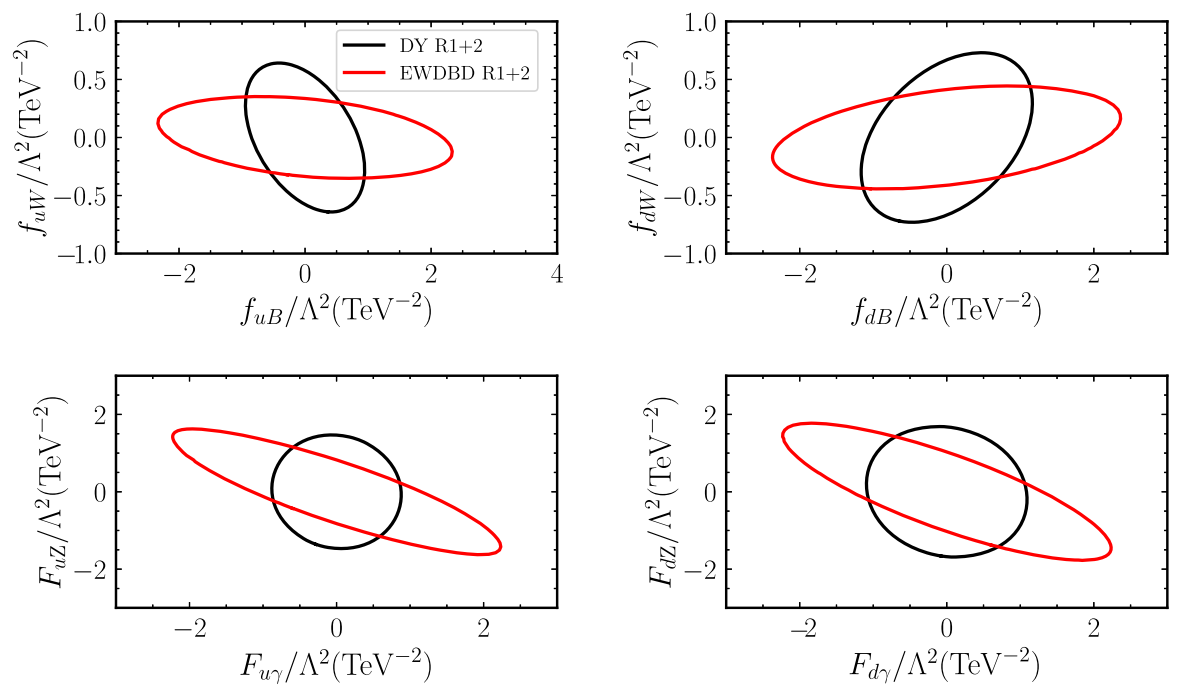

FIG. 4. $95 \%$ allowed regions from the combined EWDBD analysis (red lines) and the combined DY data analysis (black lines) in the planes $f_{q W} / \Lambda^{2}$ vs $f_{q B} / \Lambda^{2}$ and $F_{q Z} / \Lambda^{2}$ vs $F_{q} \gamma / \Lambda^{2}$.

to a mild energy growth in the corresponding helicity amplitudes $q \bar{q} \rightarrow \ell^{-} \ell^{+}$, which at high energy behave as

$$
\begin{aligned}
& A\left(q_{+} \bar{q}_{+} \rightarrow \ell_{-}^{-} \ell_{+}^{+}\right) \\
& \quad=A\left(q_{-} \bar{q}_{-} \rightarrow \ell_{-}^{-} \ell_{+}^{+}\right) \\
& \quad=\frac{e^{2} v}{\sqrt{2}} \sqrt{s} \sin \theta\left[-\frac{F_{q \gamma}}{\Lambda^{2}}+\frac{1}{c_{W} s_{W}}\left(-\frac{1}{2}+s_{W}^{2}\right) \frac{F_{q Z}}{\Lambda^{2}}\right],
\end{aligned}
$$

$$
\begin{aligned}
A\left(q_{+} \bar{q}_{+} \rightarrow \ell_{+}^{-} \ell_{-}^{+}\right) & =A\left(q_{-} \bar{q}_{-} \rightarrow \ell_{+}^{-} \ell_{-}^{+}\right) \\
& =\frac{e^{2} v}{\sqrt{2}} \sqrt{s} \sin \theta\left[\frac{F_{q \gamma}}{\Lambda^{2}}-\frac{s_{W}}{c_{W}} \frac{F_{q Z}}{\Lambda^{2}}\right]
\end{aligned}
$$

where $\theta$ is the center-of-mass polar scattering angle.

Both ATLAS and CMS have published in Refs. [44,45], respectively, the final results of the Run 1 Drell-Yan measurements in the form of differential Drell-Yan cross section as a function of the invariant mass of the lepton pair after correction for detector effects and also giving a very detailed account of the systematic and theoretical uncertainties after the unfolding of detector effects. These data allow us a very straightforward comparison with the invariant-mass differential cross section predictions including the effect of the dipole couplings.

As for Run 2, CMS has also presented the corresponding differential cross section results but only for a small integrated luminosity [46]. However, both collaborations performed a search for high-mass phenomena in dilepton final states using larger Run 2 samples [47,48]. These data can also be analyzed to study the effect of dipole operators. In this case, we follow a procedure similar to that sketched in Sec. III for the analysis of EWDBD. We simulate the $e^{+} e^{-}$and $\mu^{+} \mu^{-}$invariant-mass differential distributions within the cuts of the experimental searches using the packages MADGRAPH5 [35], PYTHIA6.4 [38] and DELPHES [39]. The SM predictions from this procedure are then normalized bin by bin to the predictions provided by the experimental collaborations, and the obtained

\begin{tabular}{|c|c|c|c|}
\hline & $\begin{array}{c}\text { Integrated } \\
\text { Luminosity }\left(\mathrm{fb}^{-1}\right)\end{array}$ & $m_{\ell \ell}$ & $\begin{array}{l}\text { Number of } \\
\text { data points }\end{array}$ \\
\hline $\begin{array}{l}\text { ATLAS } \\
13 \mathrm{TeV} \text { [47] }\end{array}$ & $36 \mathrm{fb}^{-1}$ & $250-6000 \mathrm{GeV}$ & $6+6$ \\
\hline $\begin{array}{l}\text { CMS } \\
\quad 13 \mathrm{TeV} \text { [48] }\end{array}$ & $36 \mathrm{fb}^{-1}$ & $200-3000 \mathrm{GeV}$ & $6+6$ \\
\hline $\begin{array}{l}\text { ATLAS } \\
\quad 8 \mathrm{TeV} \text { [44] }\end{array}$ & $20.3 \mathrm{fb}^{-1}$ & $200-1500 \mathrm{GeV}$ & 8 \\
\hline $\begin{array}{l}\text { CMS } \\
\quad 8 \mathrm{TeV}\end{array}$ & $19.7 \mathrm{fb}^{-1}$ & 200-2000 GeV & 11 \\
\hline
\end{tabular}
correction factors are subsequently applied to the predicted distributions in the presence of the dipole operators.

In summary, we include the data samples in our DrellYan analysis, ${ }^{1}$

and with those and the information provided by the experiments on the systematic and theoretical uncertainties and correlations, we build a binned log-likelihood function,

$$
\chi_{\mathrm{DY}}^{2}\left(f_{u B}, f_{u W}, f_{d B}, f_{d W}\right)
$$

where for simplicity we have set to zero the coefficients of all other operators contributing to the process.

\footnotetext{
${ }^{1}$ We only consider in the analysis the bins with invariant mass above approximately $200 \mathrm{GeV}$ where the dipole operator contribution is potentially more relevant. Also, for better statistical significance, we have combined in one bin the data for the last three invariant-mass bins in Ref. [47].
} 
As discussed in the previous section, the amplitudes generated by dipole operators do not interfere with the SM ones nor with those generated by the other dimension- 6 operators in Eq. (2.11), and therefore no cancellation of their effects is possible. So, as it was the case in the analysis of EWDBD and of EWPD, the constraints from DY on the Wilson coefficients of dipole operators can only be marginally affected by the inclusion of other operators in the analysis.

The results for the DY analysis are depicted in Fig. 5, in which we display one-dimensional projections of the $\Delta \chi_{\mathrm{DY}}^{2}$ as a function of the Wilson coefficient of each dipole operator after marginalizing over the other three. We show the results using each of the data samples separately and the combination. As seen in the figure, the constraints imposed with the analysis of the ATLAS 8 Run 1 DY results are substantially stronger than those obtained from the analysis of the corresponding CMS Run 1 data.

We trace this difference to the fact that the ATLAS results are slightly lower than the SM predictions in all bins included in the analysis (see Table 12 in Ref. [49]), which results in bounds that are about two times stronger than the expected sensitivity from data centered in the SM predictions. On the contrary, CMS finds a mild excess of events with respect to the SM predictions for invariant

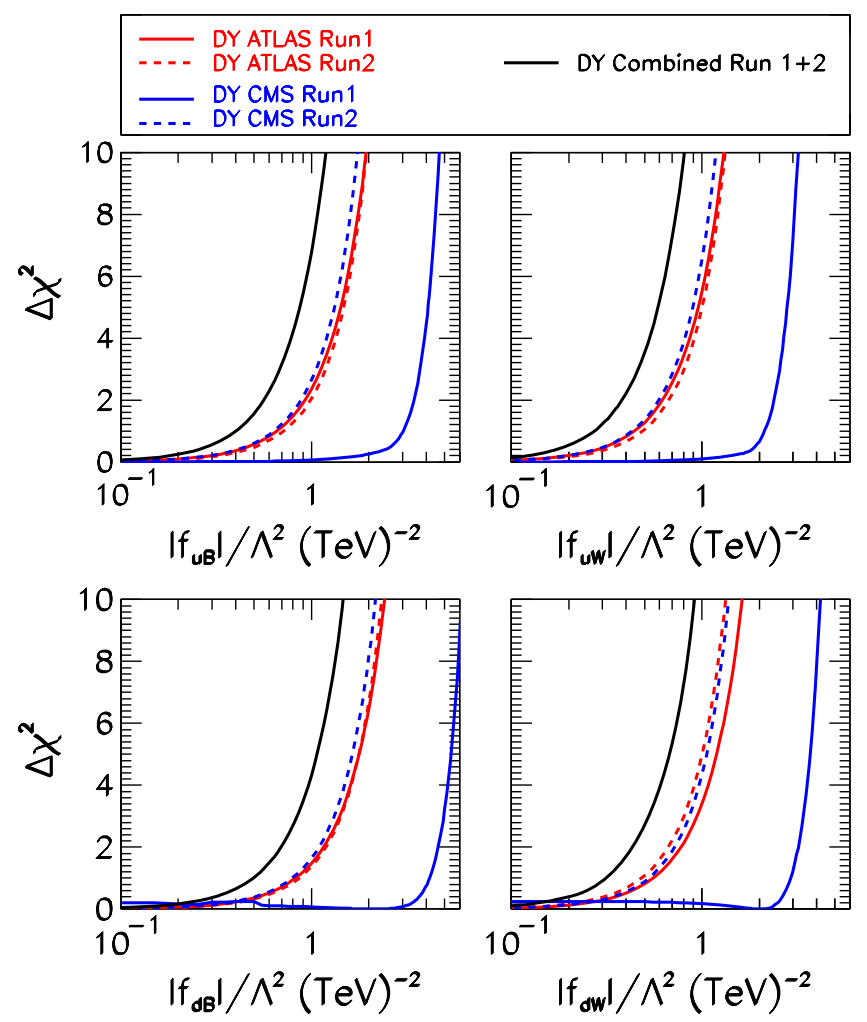

FIG. 5. $\Delta \chi^{2}$ dependence on the four fermionic Wilson coefficients of the dipole operators for the analysis of DY data (see the text for details). masses between 200 and $500 \mathrm{GeV}$ where the data are most precise (see Fig. 3 in Ref. [45]). This weakens their constraints by about $20 \%$. The analysis of the Run 2 data yields bounds very much within the expected sensitivity from measurements compatible with the SM.

Comparing the results in Fig. 5 with those from EWDBD analysis in Fig. 1, we find that the combined analysis of the Drell-Yan data can yield slightly stronger (weaker) bounds on the coefficients of the quark dipole operators $\mathcal{O}_{q B}\left(\mathcal{O}_{q W}\right)$. However, as seen in Fig. 4, DY results totally resolve the light-quark dipole couplings to $Z$ and $\gamma$ and, consequently, yield stronger constraints over those projections.

\section{DISCUSSION}

In this work, we have studied the power of the highenergy LHC data to reveal the effects associated to electroweak dipole couplings of the light quarks. We have focused on two types of processes: pair production of electroweak gauge bosons and Drell-Yan lepton pair production. Because of their different tensor structure, the amplitudes induced by these couplings do not interfere with the SM ones nor with those generated by the other dimension- 6 operators that modify the gauge-boson couplings to fermions and TGCs. Consequently, we find that the constraints derived on all the Wilson coefficients of those nondipole operators entering the tests of the electroweak gauge-boson sector are robust under the inclusion of the light-quark dipole operators.

Dipole couplings of the light quarks to the weak gauge bosons have been explored in the past using the precise data of the on-shell $Z$ and $W$ couplings to fermions. Our results show that analyses of LHC data improve over those by more than 1 order of magnitude. This is explicitly quantified in Table I, in which we contrast the resulting constraints from the analysis of the data on EWDBD and DY at LHC with those from the pole measurements. The improvements in the constraints are due to the growth

TABLE I. Comparison of the $95 \%$ upper bounds for the Wilson coefficients of the light-quark dipole operators for the different analysis performed in this work.

\begin{tabular}{lccc}
\hline \hline & \multicolumn{3}{c}{$95 \%$ C.L. $|f| / \Lambda^{2}\left(\mathrm{TeV}^{-2}\right)$} \\
\cline { 2 - 4 } & EWPD & EWDBD + EWPD & DY \\
\hline$f_{u B}$ & 41 & 1.9 & 0.78 \\
$f_{u W}$ & 10 & 0.29 & 0.53 \\
$f_{d B}$ & 38 & 1.9 & 0.96 \\
$f_{d W}$ & 10 & 0.36 & 0.60 \\
$F_{u \gamma}$ & 51 & 1.8 & 0.78 \\
$F_{u Z}$ & 7.0 & 1.3 & 1.2 \\
$F_{d \gamma}$ & 48 & 1.8 & 0.91 \\
$F_{d Z}$ & 5.8 & 1.4 & 1.4 \\
\hline \hline
\end{tabular}


of the dipole contribution with energy, as well as the similar sensitivity of the LHC data to the dipole couplings to $Z$ and $\gamma$ s. Consequently, as seen in this table, the LHC bounds on the combinations entering the dipole couplings to the $Z$ and the photon are comparable.

It is important to stress that the constraints derived with LHC data are obtained in the asymptotic free regime for the light quarks. So, in this respect, the information provided by LHC complements the more model-dependent limits on the dipole couplings of the light quarks, which can be derived from measurements of the anomalous magnetic moments of hadrons [50].

\section{ACKNOWLEDGMENTS}

This work is supported in part by Conselho Nacional de Desenvolvimento Científico e Tecnológico (CNPq) and by Fundação de Amparo à Pesquisa do Estado de São Paulo (FAPESP) Grant No. 2018/16921-1, by U.S. NSF Grant No. PHY-1620628, by EU Networks FP10 ITN ELUSIVES (Grant No. H2020-MSCA-ITN-2015-674896) and INVISIBLES-PLUS (Grant No. H2020-MSCARISE-2015-690575), by Ministry of Economy and Competitiveness (MINECO) Grant No. FPA2016-76005C2-1-P, and by Maria de Maetzu program Grant No. MDM2014-0367 of Institute of Cosmos Sciences.
[1] G. Aad et al. (ATLAS Collaboration), Phys. Lett. B 716, 1 (2012).

[2] S. Chatrchyan et al. (CMS Collaboration), Phys. Lett. B 716, 30 (2012).

[3] S. Weinberg, Physica (Amsterdam) 96A, 327 (1979).

[4] W. Buchmuller and D. Wyler, Nucl. Phys. B268, 621 (1986).

[5] B. Grzadkowski, M. Iskrzynski, M. Misiak, and J. Rosiek, J. High Energy Phys. 10 (2010) 085.

[6] Z. Zhang, Phys. Rev. Lett. 118, 011803 (2017).

[7] J. Baglio, S. Dawson, and I. M. Lewis, Phys. Rev. D 96, 073003 (2017).

[8] A. Alves, N. Rosa-Agostinho, O. J. P. Éboli, and M. C. Gonzalez-Garcia, Phys. Rev. D 98, 013006 (2018).

[9] E. da Silva Almeida, A. Alves, N. Rosa Agostinho, O. J. P. Éboli, and M. C. Gonzalez-Garcia, Phys. Rev. D 99, 033001 (2019).

[10] J. Baglio, S. Dawson, and I. M. Lewis, Phys. Rev. D 99, 035029 (2019).

[11] R. Escribano and E. Masso, Nucl. Phys. B429, 19 (1994).

[12] G. Kopp, D. Schaile, M. Spira, and P. M. Zerwas, Z. Phys. C 65, 545 (1995).

[13] S. Alioli, W. Dekens, M. Girard, and E. Mereghetti, J. High Energy Phys. 08 (2018) 205.

[14] A. Buckley, C. Englert, J. Ferrando, D. J. Miller, L. Moore, M. Russell, and C. D. White, J. High Energy Phys. 04 (2016) 015.

[15] N. P. Hartland, F. Maltoni, E. R. Nocera, J. Rojo, E. Slade, E. Vryonidou, and C. Zhang, J. High Energy Phys. 04 (2019) 100.

[16] H. D. Politzer, Nucl. Phys. B172, 349 (1980).

[17] H. Georgi, Nucl. Phys. B361, 339 (1991).

[18] C. Arzt, Phys. Lett. B 342, 189 (1995).

[19] H. Simma, Z. Phys. C 61, 67 (1994).

[20] K. Hagiwara, S. Ishihara, R. Szalapski, and D. Zeppenfeld, Phys. Rev. D 48, 2182 (1993).

[21] K. Hagiwara, T. Hatsukano, S. Ishihara, and R. Szalapski, Nucl. Phys. B496, 66 (1997).

[22] A. De Rujula, M. B. Gavela, P. Hernandez, and E. Masso, Nucl. Phys. B384, 3 (1992).
[23] J. Elias-Miro, J. R. Espinosa, E. Masso, and A. Pomarol, J. High Energy Phys. 11 (2013) 066.

[24] T. Corbett, O. J. P. Éboli, J. González-Fraile, and M. C. González-Garcia, Phys. Rev. D 87, 015022 (2013).

[25] T. Corbett, O. J. P. Éboli, and M. C. Gonzalez-Garcia, Phys. Rev. D 91, 035014 (2015).

[26] T. Corbett, O. J. P. Éboli, and M. C. Gonzalez-Garcia, Phys. Rev. D 96, 035006 (2017).

[27] LEP, ALEPH, DELPHI, L3, and OPAL Collaborations, and LEP and TGC Working Group, A combination of preliminary results on gauge boson couplings measured by the LEP experiments, http://lepewwg.web.cern.ch/ LEPEWWG/lepww/tgc, 1EPEWWG/TGC/2002-02.

[28] G. Aad et al. (ATLAS Collaboration), J. High Energy Phys. 09 (2016) 029.

[29] V. Khachatryan et al. (CMS Collaboration), Eur. Phys. J. C 76, 401 (2016).

[30] G. Aad et al. (ATLAS Collaboration), Phys. Rev. D 93, 092004 (2016).

[31] V. Khachatryan et al. (CMS Collaboration), Eur. Phys. J. C 77, 236 (2017).

[32] ATLAS Collaboration, CERN Report No. ATLAS-CONF2016-043, https://cds.cern.ch/record/2206093.

[33] ATLAS Collaboration, CERN Report No. ATLAS-CONF2018-034, https://cds.cern.ch/record/2630187.

[34] M. Aaboud et al. (ATLAS Collaboration), Eur. Phys. J. C 78, 24 (2018).

[35] J. Alwall, R. Frederix, S. Frixione, V. Hirschi, F. Maltoni, O. Mattelaer, H. S. Shao, T. Stelzer, P. Torrielli, and M. Zaro, J. High Energy Phys. 07 (2014) 079.

[36] N. D. Christensen and C. Duhr, Comput. Phys. Commun. 180, 1614 (2009).

[37] A. Alloul, N. D. Christensen, C. Degrande, C. Duhr, and B. Fuks, Comput. Phys. Commun. 185, 2250 (2014).

[38] T. Sjostrand, S. Mrenna, and P. Z. Skands, J. High Energy Phys. 05 (2006) 026.

[39] J. de Favereau, C. Delaere, P. Demin, A. Giammanco, V. Lemaitre, A. Mertens, and M. Selvaggi (DELPHES 3 Collaborations), J. High Energy Phys. 02 (2014) 057. 
[40] S. Schael et al. (DELPHI, ALEPH, SLD, OPAL, and L3 Collaborations, SLD Heavy Flavour, SLD Electroweak, and LEP Electroweak Working Group), Phys. Rep. 427, 257 (2006).

[41] C. Patrignani et al. (Particle Data Group), Chin. Phys. C 40, 100001 (2016).

[42] L. E. W. Group (Tevatron Electroweak Working Group, SLD Electroweak and Heavy Flavour Groups, LEP Electroweak Working Group, CDF, DELPHI, ALEPH, SLD, OPAL, D0, and L3 Collaborations), arXiv:1012.2367.

[43] M. Ciuchini, E. Franco, S. Mishima, M. Pierini, L. Reina, and L. Silvestrini, Nucl. Part. phys. Proc. 273, 2219 (2016).

[44] G. Aad et al. (ATLAS Collaboration), J. High Energy Phys. 08 (2016) 009.
[45] V. Khachatryan et al. (CMS Collaboration), Eur. Phys. J. C 75, 147 (2015).

[46] A. M. Sirunyan et al. (CMS Collaboration), arXiv:1812. 10529.

[47] M. Aaboud et al. (ATLAS Collaboration), J. High Energy Phys. 10 (2017) 182.

[48] A. M. Sirunyan et al. (CMS Collaboration), J. High Energy Phys. 06 (2018) 120.

[49] ATLAS Collaboration, Measurement of the double-differential high-mass Drell-Yan cross section in pp collisions at $\sqrt{s}=8 \mathrm{TeV}$ with the ATLAS detector, https://atlas.web.cern .ch/Atlas/GROUPS/PHYSICS/PAPERS/STDM-2014-06/.

[50] L. Brekke and J. L. Rosner, Comments Nucl. Part. Phys. 18, 83 (1988). 